\title{
Heat transfer augmentation during water steam condensation on twisted profile tubes
}

\author{
Yu. M. Brodov, K. E. Aronson, A. Yu. Ryabchikov \\ \& M. A. Nirenstein \\ Turbine and Engines Department, Ural Federal University, Russia
}

\begin{abstract}
Some results are presented of experimental and theoretical research of hydrodynamics and heat transfer during condensation of water steam (both stationary and slowly moving) on twisted profile tubes (TPT). For a heat transfer coefficient during condensation of stationary steam on TPT two characteristic areas were observed. At small values of condensate film Reynolds numbers a TPT heat transfer coefficient can be $10-15 \%$ below that of the plain tubes depending on profile parameters. With the rise of both condensate film Reynolds number and profile parameter $h / s$ heat transfer coefficient increases up to $50 \%$ in comparison to a plain tube. During slowly moving steam condensation the TPT heat transfer coefficient increases up to $70 \%$ as compared to a plain tube.

Conducted research and industrial tests results showed that the assured effect of a heat transfer coefficient increase in TPT heat exchangers could reach for turbine condensers $15 \%$, for low cycle heaters $35-40 \%$. The heat exchangers hydraulic resistance increases by $40-70 \%$.

Keywords: hydrodynamics, heat transfer, condensation, twisted profile tubes, heat exchanger, efficiency.
\end{abstract}

\section{Hydrodynamics and heat transfer during condensation of a stationary (slowly moving) steam on TPT}

Experiments on the comparative study of condensate film hydrodynamics on twisted profile tubes (TPT) and plain tubes were carried out using cold-feed models with pigment injection both into the film and on the tube surface (in the groove and on the protrusion) in a wide range of film Reynolds numbers $\left(\operatorname{Re}_{f i l m}=G /(\mu P)\right.$, where $G$ is the condensate flow rate through the lower 
section of the tube, $\mathrm{m} / \mathrm{s} ; \mu$ - condensate dynamic viscosity, $\mathrm{kg} /(\mathrm{m} \cdot \mathrm{s}) ; P$ - tube perimeter, $\mathrm{m}$ ).

The essential differences were observed in condensate film hydrodynamics on plain and twisted profile tubes. Fig. 1 illustrates the difference. On TPT when $\mathrm{Re}_{\text {fim }}$ value changes from 20 to 40 individual waves arise on the surface of the film and their amplitude is substantially less than that of on plain tube. When injecting the pigment, the film pulling along the groove is observed as well as the film swirling. The pigment moves with the film along the helix line which angle is equal to about half the angle between the groove and the vertical. When $\mathrm{Re}_{\text {film }}$ value changes from 50 to 80 the film swirl decreases and the diffusion of the pigment occurs over the entire surface of the film. When $\mathrm{Re}_{\text {film }}$ value exceeds 100 the pigment washout occurs at the distance of $3 s$ to $5 s$ from the pigment injection point. If $\mathrm{Re}_{\text {film }}$ value exceeds 125 it occurs at $2 s$ to $3 s$ when the pigment is injected on the protrusion and at $5 s$ to $7 s$ when the pigment is injected into the groove. When Reynolds number achieves $\mathrm{Re}_{\text {film }} \approx 200$ the film pulsation arises, the pigment spreads over the entire perimeter of the tube practically from the injection point and the effect of film swirling is impossible to record. As a result of observations it was found that with decreasing of groove pitch $s$ the pigment spreading in the film is more intense. Besides, the angle of pigment vertical movement (the film swirl) increases. When applying the pigment into the profile groove its washout occurs much slower than when it is applied to the protrusion. This is possibly explained by the condensate film pulling into the groove due to surface tension forces. These observations formed the basis for the formulation of an analytical model of hydrodynamics and heat transfer during steam condensation on TPT.

Fig. 2 shows the coordinate system which was employed to describe the liquid film flow on a vertical TPT.
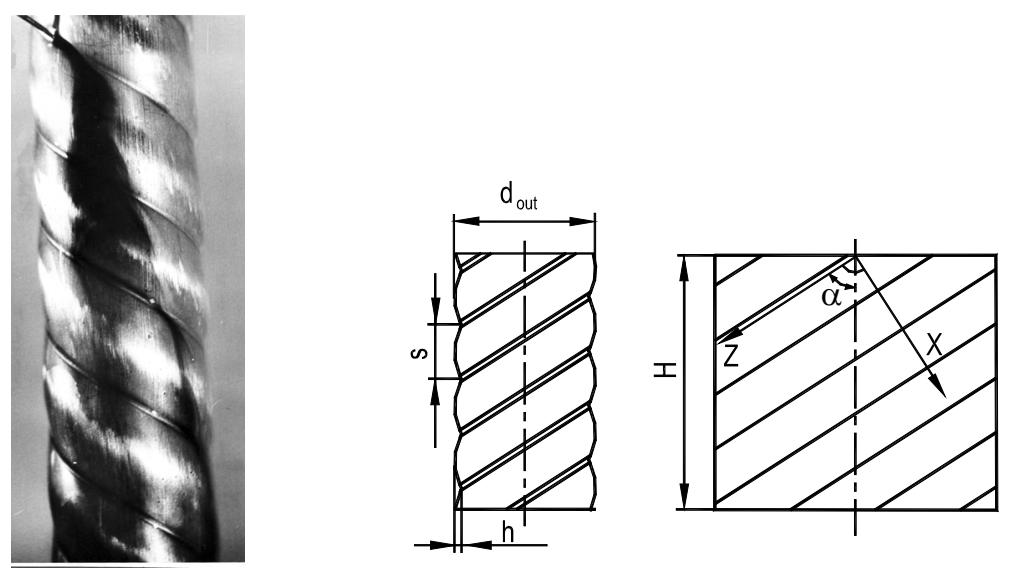

Figure 1: Condensate film Figure 2: Coordinate system on a vertical hydrodynamics on TPT. a vertical TPT 
The equations of the film motion in the moving coordinate system $\left(X_{*}, Y_{*}, Z_{*}\right)$ run as follows (Brodov et al. [1]):

$$
\left\{\begin{array}{l}
-\frac{d P}{d X_{*}}+\mu \frac{\partial^{2} u_{x *}}{\partial Y_{*}^{2}}+\rho g \sin \alpha \cdot \cos \left(\hat{X_{*} X}\right)=0 \\
\mu \frac{\partial^{2} u_{z *}}{\partial Y_{*}^{2}}+\rho g \cos \alpha=0
\end{array},\right.
$$

where $\rho$ and $u$ are condensate density, $\mathrm{kg} / \mathrm{m}^{3}$, and velocity, $\mathrm{m} / \mathrm{s}$, correspondingly; $\alpha$ is an angle of helix profile inclination.

An expression for film thickness calculation in a dimensionless form is taken on the basis of eqn (1) and of thermal balance equation.

$$
\frac{1}{3} \mathrm{Ga} \operatorname{Pr} \mathrm{K}\left\{\frac{\partial}{\partial x_{1}}\left[\left(\sin \alpha \cos \left(x_{1}^{\wedge} x\right)-\mathrm{We} \frac{\partial}{\partial x_{1}}\left(\frac{1}{R_{\text {film }}^{\prime}}\right)\right) \delta_{1}^{3}\right]+\frac{\partial}{\partial z_{1}}\left(\delta_{1}^{3} \cos \alpha\right)\right\} \delta_{1}=1
$$

where $\mathrm{Ga}$ is Galilei number, $\mathrm{Pr}$ is Prandtl number, $\mathrm{K}=r /\left(c_{\mathrm{p}} \Delta T\right)$ is Kutateladze (phase-to-phase transition) number; We is Weber number; $\delta_{1}=\delta / H$ is dimensionless film thickness; $R_{\text {film }}^{\prime}=R_{\text {film }} / H$ - dimensionless curvature radius of condensate film surface; $x_{1}=X_{*} / H, z_{1}=Z_{*} / H$ - dimensionless linear variables; $\delta$ is the condensate film thickness, $\mathrm{m} ; H$ is the tube height, $\mathrm{m}$.

For eqns (1) and (2) the following assumptions are used:

1. condensate film thickness is small in comparison to TPT geometrical characteristics $\left(d_{\text {out }}, s, h\right)$;

2. heat conductivity along the film is considerably less than heat conductivity across it and heat convection in the film is also small;

3. friction is absent on the surface between liquid and vapor phases;

4. the temperature on the film outer surface is equal to steam saturation temperature and the wall temperature along the surface of condensation is constant;

5. the changes of condensate thermal physical properties across the film are not taken into account, condensate physical properties are calculated according to steam saturation temperature;

6. the density of steam is small as compared to the condensate one;

7. condensate depression is not taken into account;

8. condensate film flow is laminar, inertia forces are negligible as compared to density forces.

The structure of eqn (2) includes physical parameters determining the process and parameter of the film dimensionless curvature that makes it possible to calculate the relative film thickness at a certain section of the vertical TPT. As $s \rightarrow \infty$ and $h=0$ the eqn (2) converts to the classic equation for a plain vertical tube (Isachenko [2]). 


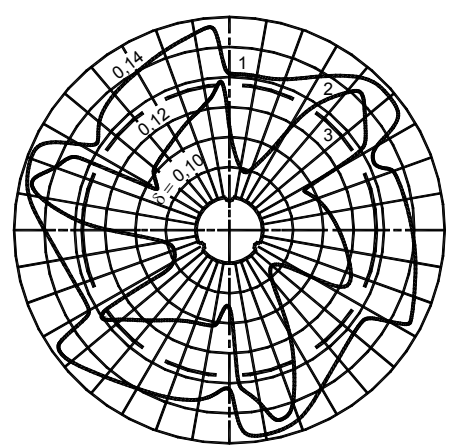

Figure 3: The diagram of condensate film thickness distribution along the perimeter of the vertical plain tube and TPT. $(H=0.706 \mathrm{~mm}$; $\Delta T=10^{\circ} \mathrm{C} ; 1-h=0.3 \mathrm{~mm} ; s=15 \mathrm{~mm} ; 2-0.6 / 15 ; 3-$ plain tube.)

Eqn (2) was solved using a numerical procedure. As a first approximation it was assumed that the film surface curvature was equidistant to the TPT surface curvature. The calculations were done using a computational grid with the width changing from 6 to 10 degrees and the height - from 1 to $2 \mathrm{~mm}$.

In fig. 3, as an example, the results are presented of calculations by eqn (2) the characteristic diagrams of the condensate film thickness variation along the perimeter of the vertical plain tube and TPT. The film thickness is minimal in the transition zone from the protrusion to the groove and in the middle of the protrusion.

\section{Experimental study of heat transfer during steam condensation on TPT}

Heat transfer research of condensation of a stationary (slowly moving) steam was carried out on experimental setup (fig. 4) using a local heat modeling method.

The tube wall temperature is taken by tube electrical resistance measuring on the basis of previously obtained calibration curves (the tube material was stainless steel). Such process for wall temperature measuring in comparison with other known methods has several advantages: the temperature is determined as average over the perimeter, height and thickness of the tube wall; the heat transfer surface is not affected which is particularly important in experiments with steam condensation on the tubes outer surface.

Systematic instrumental mean-square uncertainties for main measured parameters at a probability value of 0.95 are as follows: water flow rate $-0.1 \%$, water temperature differences - from 0.1 to $2.0 \%$, "steam-tube wall" temperature difference - from 2.9 to $5.4 \%$. The maximum mean-square error of the heat transfer coefficient does not exceed \pm 6 .

The results for the relative heat transfer during condensation of a stationary (slowly moving) steam on vertical TPT are shown on fig. 5. Analysis of experimental data showed that the relative heat transfer coefficient during 
condensation of steam on TPT $\left(\mathrm{Nu}_{\mathrm{TPT}}^{\circ} / \mathrm{Nu}_{p l}^{\circ}\right)$ depends mainly on condensate film flow regime, TPT groove pitch and depth values and is generalized by the following expression:

$$
\frac{\mathrm{Nu}_{\mathrm{TPT}}^{\mathrm{o}}}{\mathrm{Nu}_{p l}^{\mathrm{o}}}=\operatorname{Re}_{f i l m}^{a}\left(\frac{h}{s}\right)^{b},
$$

where $\mathrm{Re}_{\text {film }}=80-400 ; a=0.133 ; b=0.25$.

The average deviation of experimental and calculated by (3) data is no more than $10 \%$.

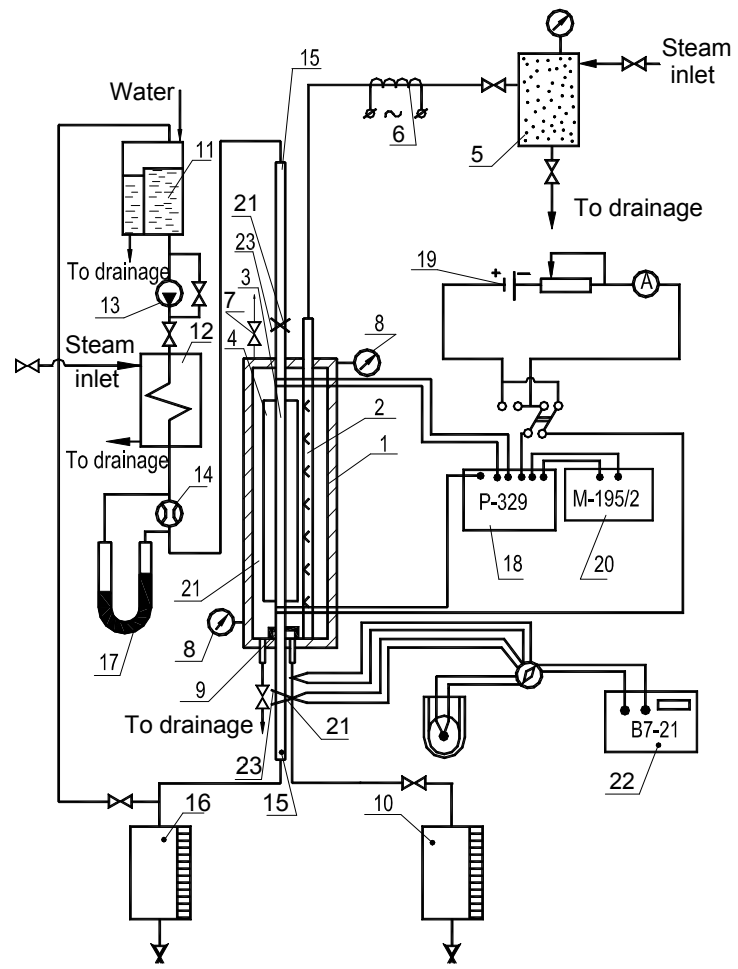

Figure 4: Experimental setup for stationary steam condensation research on vertical tubes. ( 1 - test chamber, 2 - steam supply, 3 - test tube, 4 - inspection windows, 5- steam receiver, 6 - electric superheater, 7 - blowdown valve, 8 - calibration manometer, 9 - condensate receptacle, 10 - condensate measuring tank, 11 - pressure tank, 12 - auxiliary heater, 13 - pump, 14 - orifice plate, 15 - hydrodynamic stabilization section, 16 - measuring tank, 17 - differential manometer, 18 - resistance meter P-329, 19 - power supply, 20 - mirror galvanometer M-195/2, 21 - Chromel-Copel thermocouples, 22 - digital voltmeter B7-21, 23 - differential thermocouples.) 
Studies have shown that the heat transfer coefficient during condensation of slowly moving steam on TPT can be either lower or higher than the coefficient of heat transfer on a plain tube. The maximum reduction of heat transfer coefficient in the investigated range of Reynolds numbers and TPT profile parameters rises up to $12 \%$ (see fig. 5). With the increase of $\operatorname{Re}_{\text {film }}$ and $h / s$ parameter the intensity of heat transfer also rises, the maximum increase being of $50 \%$. The experimental results can be explained on the basis of observations of film hydrodynamics on the surface of twisted profiled tubes.

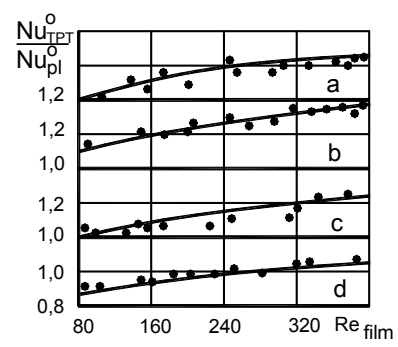

Figure 5: Heat transfer during condensation of a stationary steam on TPT. $(--$ calculation; $\bullet-$ experiment data; $\mathrm{a}-h / s=0.2 ; \mathrm{b}-0.147$; $c-0.094 ; d-0.048$.)

Thus at relatively shallow grooves $(h<0.4 \mathrm{~mm})$ the intensity of film swirling on TPT surface is affected, apparently, by knurling pitch while the action of surface tension forces leading to a change in film thickness around the tube perimeter is weak. The length of the film flow path on TPT surface as compared to that of the plain tube (in case of strictly vertical flow) increases and, consequently, there is a greater amount of fluid on the surface of the tube per time unit that determines the deterioration of heat transfer from the steam. As the groove depth value is increasing (even with an increase of knurling pitch $s$ ) the surface tension forces begin to play a more active role thus significantly changing the film thickness around the tube perimeter which causes activation of the film pulling into the groove and a decrease in the average film thickness at protrusions (due to film pulling into the groove). As a result the average film thickness decreases around the perimeter of the TPT (in comparison with plain tube) and, therefore, steam heat transfer coefficient increases.

Comparison of experimental data with calculations based on model (2) shows a qualitative agreement (fig. 6). As the tube diameter affects heat transfer coefficient in accordance with mathematical model, parameter $h / s^{*}$ was chosen to be a criterion characterizing the TPT geometry. Marked in the fig. 6 ranges of experimental data for each of the investigated TPT (with $h / s^{*}=$ const) correspond to different flow regimes of the condensate film. As it can be seen from fig. 6, the experimental data qualitatively confirm the results of calculations by the analytical model and show that under certain knurling parameters both augmentation and deterioration of heat transfer is possible in comparison with plain tube. The quantitative divergence of the experimental and calculated data 
can be explained by some restrictions imposed on the model as well as by a significant influence of the condensate film flow regime which is not taken into account in the model.

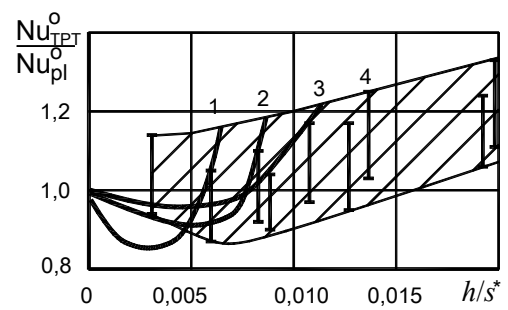

Figure 6: Heat transfer during condensation of a stationary steam on a vertical TPT. $1,2,3-s=2,15,32 \mathrm{~mm} ; 4$ - range of experimental data for each TPT; cross-hatching corresponds to experimental data field.

\section{Condensation of moving steam on TPT}

Experimental study of hydrodynamics and heat transfer during condensation of steam cross flow on vertical TPT was conducted using a local modeling method. The experimental setup is shown in fig. 7 (Berg et al. [3]).

Fluid temperatures were measured by calibrated Chromel-Copel thermocouples and controlled by laboratory thermometers with a scale division $0.1^{\circ} \mathrm{C}$. The wall temperature of tested tubes (material - stainless steel) was determined from measurements of tube electrical resistance according to the previously obtained calibration curves. Cooling water flow rate was measured by volumetric method. Steam flow velocity was determined by thermoelectric velocity sensor (Berg et al. [3]).

Knurling parameters of investigated TPT were as follows: groove pitch $s-$ from 7.0 to $19.0 \mathrm{~mm}$, groove depth $h$ - from 0.4 to $1.2 \mathrm{~mm}$, number of starts $z$ 1 and 3. Mean-square uncertainties did not exceed for steam velocity measurement $\pm 6 \%$, for heat transfer coefficient determination $\pm 8 \%$.

The experiments were conducted at steam pressure from 0.11 to $0.12 \mathrm{MPa}$ and steam temperature was $2-3^{\circ} \mathrm{C}$ above the saturation. Volume air content in the steam did not exceed $0.01 \%$. Temperature drop from steam to tube wall varied from 5 to $50^{\circ} \mathrm{C}$. The velocities of the incoming steam flow were from 0.5 to $4.5 \mathrm{~m} / \mathrm{s}$ and that corresponds to steam velocity in the narrow section of the channel from 1.5 to $20.0 \mathrm{~m} / \mathrm{s}$.

Visual observations showed that during the condensation of moving steam cross flow the vertical plain tube perimeter can be divided into two characteristic zones of film flow - a front zone and a tail one (fig. 8a). Under the influence of approach steam flow the film moves from the frontal to the tail zone. The frontal zone film thickness along the tube vertical generatrix is approximately constant. In lateral areas of the tube the film thickness apparently increases which could be caused by steam boundary layer separation from the tube surface and the formation of steam reverse flow behind the separation point. 


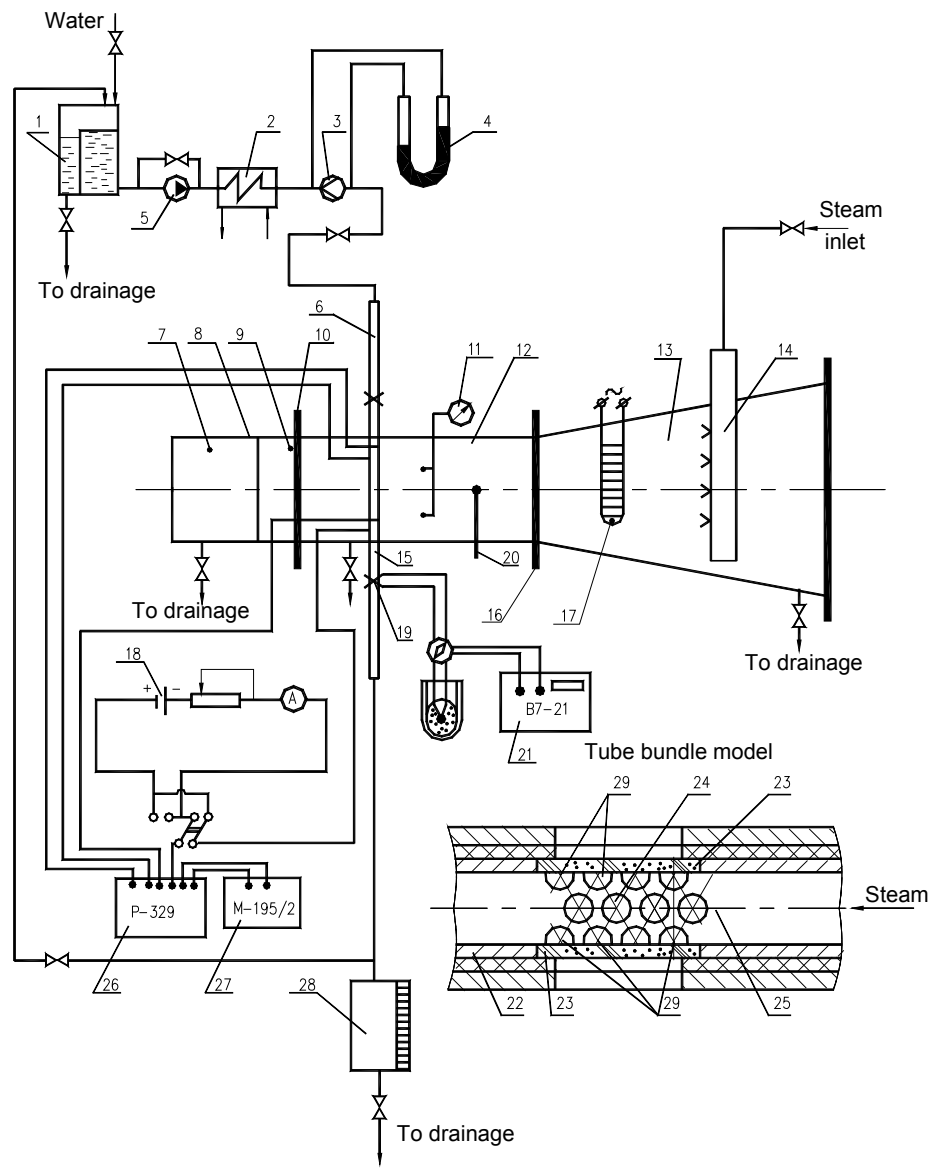

Figure 7: Experimental setup for moving steam condensation research. ( 1 - pressure tank; 2 - water heater; 3 - orifice plate; 4 -differential manometer; 5 - pump; 6 - hydrodynamic stabilization section; 7- expander; 8 - exhaust part; 9 - channel; 10, 16 - flanges; 11 - manometer; 12 - test chamber; 13 - converging receiver; 14 - steam supply collector; 15,24 - test tube; 17 - superheater; 18 - power supply; 19 - thermocouples; 20 - thermoelectric velocity measuring sensor; 21 - voltmeter; 22 - channel wall; 23 - inspection window; 25 - test channel; 26 - resistance meter P329; 27 - galvanometer; 28 - measuring tank; 29 - tubes of the tube bundle model.)

In the tail area the film is not exposed to dynamic effect of the steam flux and flows along the generatrix of the tube downward. There is an area of active wave formation on the film surface at the tail zone which is caused by increased hydraulic rating due to the bulk flow of condensate on the part of the tube 
surface (about half the perimeter). Condensate film profile along the plain tube perimeter is symmetric to the direction of the incident steam flux.

During the condensation of approach steam flow on TPT the condensate film is redistributed along the tube perimeter in a manner similar to plain tube one. However, in contrast to the plain tube, the film profile is asymmetrical to the direction of the incident flow (fig. 8(b)). A film displacement is observed from the symmetry position to the direction of the helix profile line (top to bottom) which leads to a decrease of tail zone area (in comparison with plain tube). The latter apparently is determined by the fact that the incident steam flow at one side of the tube actively prevents the displacement of a thickened part of the film from the tail to the frontal zone (fragment I, fig. 8(b)). On the opposite side of the tube (fragment II, fig. 8(b)) the incident steam flux facilitates the movement of the film in the direction of the tube helix profile line. In the tail zone of TPT the film practically is not affected by the dynamic impact of steam which is quite similar to a plain tube. Experimental data on heat-transfer coefficient ratio during condensation of moving steam on a number of vertical TPT in comparison with that of on plain tubes are plotted in fig. 9 in the coordinates of $\mathrm{Nu}_{\mathrm{Tr}}^{w} / \mathrm{Nu}_{p l}^{\mathrm{o}}=f\left(\Pi_{1}\right)$.

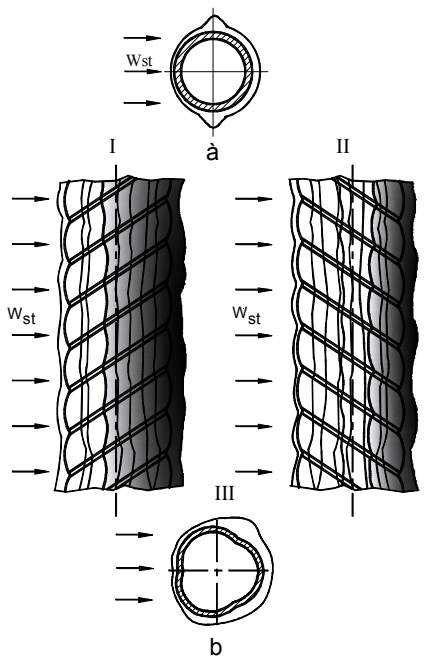

Figure 8: Film hydrodynamics during moving steam condensation on vertical plain (a) and twisted profiled (b) tubes.

For TPTs a new complex $\Pi_{1}$ similar to complex $\Pi$ for plain tubes was used as a defining parameter $\left(\Pi=\frac{\mathrm{Fr}_{s t}}{\operatorname{Pr}_{f} \mathrm{~K}} \cdot \frac{H}{d_{\text {out }}} ; \quad \Pi_{1}=\frac{\mathrm{Fr}_{s t}}{\operatorname{Pr}_{f} \mathrm{~K}^{0.2}} \cdot \frac{H}{d_{\text {out }}}\right)$ (Brodov et al. [4]); here $\mathrm{K}=r /\left(c_{\mathrm{p}} \Delta T\right)$ is Kutateladze (phase-to-phase transition) number and $\mathrm{Fr}$ is Froude number. The experimental data processing revealed the effect of 
"steam-wall" temperature drop to be different from that of plain tube. In connection with this the power of Kutateladze number $\mathrm{K}$ changed from $(-1)$ in $\Pi$ to $(-0.2)$ in $\Pi_{1}$.

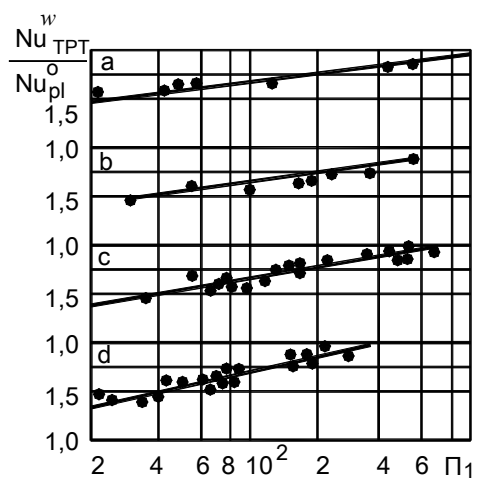

Figure 9: Heat transfer on vertical TPT during condensation of transverse steam flow. - - calculation by eqn (4); $\bullet$ - experimental data; $\mathrm{a}-h / \mathrm{s} / d_{\text {out }}=0.4 / 7.0 / 16.0 \mathrm{~mm} ; \mathrm{b}-0.7 / 7.0 / 16.0 \mathrm{~mm} ; \mathrm{c}-0.8 / 12.0 /$ $19.0 \mathrm{~mm} ; \mathrm{d}-1.2 / 19.0 / 19.0 \mathrm{~mm}$.

The relative effect of heat transfer on TPT is affected both by the process parameters and by the parameters of tube profile. Experimental data for heat transfer coefficient during condensation of transverse water steam flow on vertical TPT $\left(\mathrm{Nu}_{\mathrm{TPT}}^{w}\right)$ in comparison with that of a stationary (slowly moving) steam on plain tubes $\left(\mathrm{Nu}_{p l}^{\circ}\right)$ with an accuracy of $\pm 15 \%$ are summarized by an expression

$$
\frac{\mathrm{Nu}_{\mathrm{TPT}}^{w}}{\mathrm{Nu}_{p l}^{\mathrm{o}}}=\mathrm{Nu}^{*}=0.3\left(\frac{h s}{d_{\text {out }}^{2}}\right)^{-0.28} \cdot \Pi_{1}^{0.67\left(\frac{h s}{d_{\text {out }}^{2}}\right)^{-0.38}}
$$

which is valid for $14 \leq \Pi_{1} \leq 1000 ; h-$ from $0.065 s$ to $0.122 s ; s-$ from $0.438 d_{\text {out }}$ to $d_{\text {out }}$.

Comparison of experimental data for moving steam condensations on vertical TPT and on plain tubes (with steam velocity $w_{\text {st }}=i d e m$ and $\Delta T=i d e m$ ) shows that the relative effect of heat transfer for TPT $\left(\mathrm{Nu}^{*}\right)$ is from 10 to $70 \%$ higher than that of the plain tubes depending on process parameters and tube profile geometry (fig. 10).

This is probably caused by the described differences in the film hydrodynamics on the tube surface, in particular by the increase in frontal zone area of TPT in comparison to plain tube and by further enhancement of heat transfer in TPT tail zone caused by film turbulence due to artificial roughness. 


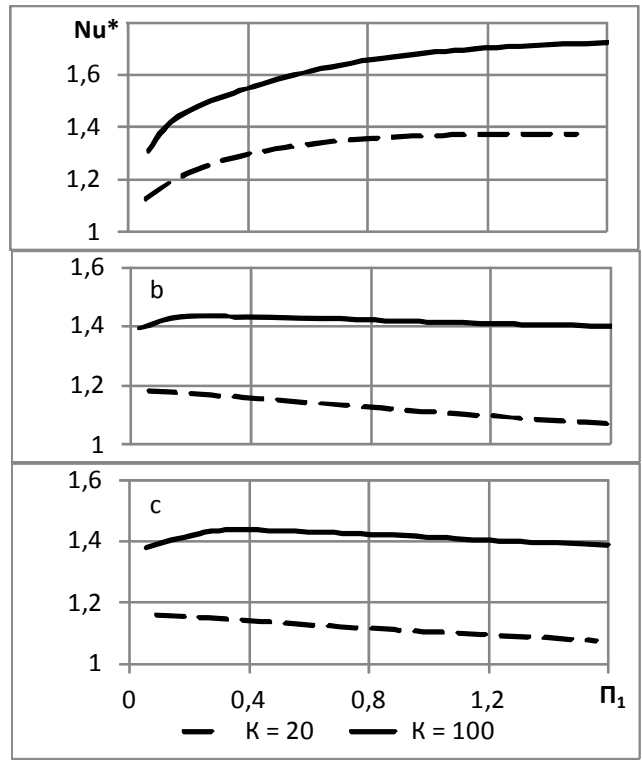

Figure 10: Heat transfer enhancement effect for moving steam condensation on TPT in comparison to plain tubes. $\left(\mathrm{a}-h / s / d_{\text {out }}=1.2 / 19.0 / 19.0 ; \mathrm{b}-\right.$ 0.7/9.0/19.0; c - 0.7/7.0/16.0.)

\section{Conclusion}

The results of steam condensation investigation for twisted profiled tubes show that condensate film flow on TPT surface is affected by gravity and surface tension forces causing the condensate film swirl and driving it to the grooves, thus decreasing the film thickness.

During condensation of stationary and slowly moving steam at small values of condensate film Reynolds numbers the TPT heat transfer coefficient can be $10-15 \%$ below than that of the plain tubes. With the rise of condensate Reynolds number the heat transfer coefficient increases up to $50 \%$ over its plain tube value depending on profile parameters.

In moving steam condensation on TPT the condensate film redistributes over tube perimeter so that its thickness is small in the front zone and major portion of condensate is accumulated in the rear zone of the tube. Heat transfer augmentation for moving steam condensation on TPT reaches $150 \%$ in comparison to stationary steam condensation on plain tubes.

On the basis of implemented research more than 500 different heat exchangers with TPT were designed, manufactured and installed in technological systems of power stations steam turbines (Aronson et al [5]). Preproduction samples of heat exchangers underwent operation tests that confirmed high level of thermal efficiency along with high reliability. The research as well as the results of steam turbines heat exchangers pilot testing showed that the guaranteed 
effect of heat transfer coefficient increase in apparatuses with TPT at nominal values of operation parameters and standard operation conditions is $15 \%$ for condensers, 35-40\% for low-pressure heaters and 20-40\% for hot water heaters, provided that TPT profile parameters are chosen rationally (Aronson et al. [5]).

\section{References}

[1] Brodov Yu. M., Gal'perin L. G., Savel'ev R. Z., Ryabchikov A. Yu. Hydrodynamics and heat transfer in film condensation of steam on vertical twisted tubes. Thermal Engineering (English translation of Teploenergetika), 34 (7), pp. 382-385, 1987.

[2] Isachenko V. P. Condensation heat transfer, Energia: Moscow, pp. 46-56, 1977.

[3] Berg B. V., Aronson K. E., Brodov Yu. M., Ryabchikov A. Yu. Condensation of steam in transverse flow to a vertical tube. Heat transfer. Soviet research, 20 (4), pp. 472-479, 1988.

[4] Brodov Yu. M., Aronson K. E., Ryabchikov A. Yu. Heat transfer with a cross flow of steam condensing on vertical tubes. Thermal Engineering (English translation of Teploenergetika), 36 (5), pp. 270-273, 1989.

[5] Aronson K. E., Brodov Yu. M., Mutovin A. T. Steam turbine heat exchangers modernization in view of operation features at various power stations. Lambert Academic Publishing: Nurnberg. 391 pp. 2011. 